\title{
The Attention Economy of Search and Web Advertisement*
}

\author{
Alexander White $^{\dagger} \quad$ Kamal Jain ${ }^{\ddagger}$ \\ Draft: September 2012 \\ (First Version May 2010)
}

\begin{abstract}
When people go on the web, they surf. Accordingly, in many circumstances, such as when people use a search engine (SE) to find a "content website" (CW), such pairs of websites are complements. Typically, both of these types of sites show advertisements to their visitors, but they do so using very different technologies. We study the incentives for such ad-funded websites with differing technologies to force their visitors to pay attention by putting up with distracting advertisements.

We show that SEs and CWs face two distinct coordination problems when designing their advertising strategies. The first is the classic problem of double marginalization among sellers of complements. The second potential problem is new: the need to efficiently allocate demands to a given user for her attention. Because of this second issue, the market for user attention exhibits surprising behavior when competition increases. In particular, heightened competition among sites of a given type (SE or CW) may cause social welfare to decrease by giving the other type of site incentive to make more inefficient demands for the users' attention.
\end{abstract}

*The first author was an intern at Microsoft Research Redmond, in 2008, during the early stages of this research. The authors wish to thank Gary Biglaiser, Marc Bourreau, Denis Charles, Eric Horvitz, Bruno Jullien, Chris Meek, Martin Peitz, Paul Seabright, Sven Seuken, Wing Suen, Thomas Tregouet and Mike Whinston for helpful comments/discussions as well as seminar audiences at EUI Florence and Hong Kong University, participants of the 2009 INFORMS Marketing Science Conference, Ann Arbor, the 2009 Télécom ParisTech and ZEW Mannheim conferences on the economics of ICT, and the 2010 IESE Conference on the Economics of Advertising and Marketing. White is especially indebted to Jacques Crémer and Glen Weyl for their support and advice. White gratefully acknowledges the financial support he received during part of this research from the Chaire Orange de l'innovation \& régulation. The authors are solely responsible for all errors.

${ }^{+}$Tsinghua University School of Economics and Management; awhite@sem.tsinghua.edu.cn

łeBay Research Labs 


\section{Introduction}

When people use the Internet, they have access to many, many websites, and they frequently pass from one to the next. Moreover, the sites are overwhelmingly available free of charge, except that they ask for a bit of the web surfers' attention, in the form of advertisement. In effect, this attention is the "price" that sites charge. This paper studies how these prices are set.

While the person browsing the web may consider "distraction" from advertising to be a price, her attention is not an ordinary currency. When sites extract this price from Internet "users", in order to make profits, they must "channel" this attention to advertisers that are willing to pay for it. Precisely how this channeling occurs is an extremely complicated issue that is not the subject of this paper ${ }^{1}$ Instead, we take as given a feature of this channeling that has been widely discussed and study its impact on the market for advertisement-based web content. This feature is the extreme variability in the profitability of showing ads on websites, both from one ad to another on the same website, and across websites. To put it another way, websites transform attention into revenue at a rate that is non-linear and that differs from one site to another.

This heterogeneity in websites' ability to profitably advertise becomes particularly noteworthy in view of the fact that people surf the web. To be more precise, the websites an Internet user visits in a particular browsing session often contribute to the pursuit of some objective that is greater than just the collection of independent visits to the sites. In such cases, it is natural to think that, from the standpoint of the user, these sites are complementary to one another2 2

A particularly common type of situation in which multiple sites are complementary are those in which an Internet user visits a search engine (SE) in order to quickly locate a "content website" (CW) that "hosts" some material the user wishes to access. This material might be, among many possible examples, a news story, a sports highlight video or a music file. Both due to the importance of such cases and thanks to their concreteness, we use as this as our primary example throughout the paper. 仙

In what follows, we develop a model of advertising to study the impact of the aforementioned issues. A set of preliminary issues, addressed in Section 2 , regards the impact on

\footnotetext{
${ }^{1}$ See Evans (2008, 2009) for overviews of the online ad intermediation industry.

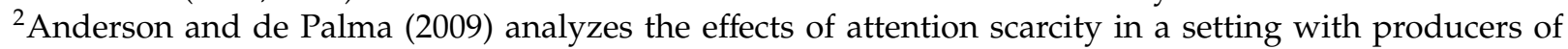
substitute goods that compete in prices.

${ }^{3}$ Despite our focus on this example, our analysis can apply to many other situations that occur on the web and may also be relevant in a variety of offline advertising environments.

${ }^{4}$ Katona and Sarvary (2008) also considers different forms of web advertising, focusing on the macro structure of the web and the impact of search engines thereupon.
} 
users of changes in advertising technology. While websites benefit from the acquisition of more efficient technologies, their effect on users is ambiguous, since such a change can lead a website to demand more of users' attention. We derive a simple condition identifying when this is the case.

When multiple websites, such as an SE and a CW, are complementary (as we assume in Sections 3 and 4), users' willingness to visit the combination of sites depends on the advertising of each. As a result, an issue for the websites is how to best coordinate their advertisement. We analyze the distortions that arise when complementary sites with different advertisement technologies choose their advertising policies independently from one another.

Section 3 considers the case where multiple complementary sites face elastic demand. We show that, in such scenarios, two distortions arise, compared to the outcome in which the sites jointly maximize profits. One distortion is the well-known phenomenon of double marginalization, uncovered in Chapter IX of Cournot (1838). When complementary sites act independently, the total distraction from their advertising is very high. As a result, the number of users that visit the set of complementary sites is lower than the number necessary to maximize total profits.

The second distortion, which we refer to as mis-marginalization, is novel to our setting. Due to their differing advertising technologies, sites inefficiently allocate the demands they make for users' attention. Consequently, holding fixed the number of users that visit the set of complementary sites, it is feasible for websites to improve upon their equilibrium level of total profits.

As a result of these two phenomena, the model has certain interesting features. On such feature is the trade-off that sites collectively face in solving the two aforementioned distortions. Typically, when there are two firms with constant marginal production costs that provide consumers with perfectly complementary components, total industry profits are optimized when one of the two firms is forced to charge a price equal to its marginal cost and the other firm is allowed to set its privately optimal price.5 However, when the price for an Internet user of visiting a site is distraction from advertisement, this property breaks down. Forcing one of the complementary sites not to advertise generally does not maximize total profits. Moreover, doing so can even cause total profits to decrease with respect to the non-cooperative outcome. While an increase in competition of this form solves the problem of double marginalization, it also exacerbates the problem of mis-marginalization.

Section 4 adapts the model to analyze the effects of differentiation among CWs and their

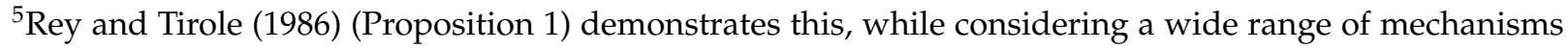
an upstream manufacturer can use to coordinate with downstream retailers.
} 
incentives to enter into the market, assuming that a single SE serves the entire set of users. Building on the framework of Salop (1979), it shows that while, under socially optimal behavior by the SE and CWs, users benefit from both decreases in differentiation among CWs and from increases in their number, under equilibrium behavior, these comparative statics are reversed. The presence of a single SE influences the outcome in such a way so that users are harmed by intense competition among CWs. This outcome results from the fact that the search engine, serving all users, always extracts all remaining rent from consumers that are indifferent between CWs. Consequently, as competition intensifies, infra-marginal users are made worse off because they, in effect, become less different from marginal ones.

Regarding the efficiency, in equilibrium, of the number of CWs, there can be either under- or over-entry, relative to the socially optimal number. When all sites behave noncooperatively and content websites have relatively inefficient advertising technologies, it is socially desirable for there to be more CWs than the number than the market induces to enter. This is because an increase in the number of CWs shifts advertising away from the CWs to the more efficient SE advertising technology.

\section{A Single Ad-Based Site}

We first consider a very simple model of the problem facing the purveyor of a single adbased website that displays some form of content users find appealing and that accrues revenue by showing advertisement. The purpose of this section is both to introduce the baseline model and to illustrate the effects of changes in advertising technology when a site raises revenue through showing users ads rather than by charging them money. The following sections then build on this model to consider cases where there are multiple sites that play a complementary role in providing users with access to content.

Suppose there is a website and a continuum of users of mass one. User $i$ receives a payoff from visiting the site given by $v_{i}-\delta(a, \gamma)$, where $v_{i}$ is the gross surplus the user derives from viewing the site's content and $\delta(a, \gamma)$ is the disutility or "distraction" the user incurs from being subjected to advertising on the site. We assume that the site can choose a method of advertising that delivers any level, $a \geq 0$, of expected revenue per visiting user, but that advertising techniques that bring about more revenue per user are also more distracting. Moreover, we assume that users' distraction accelerates as $a$ increases. The parameter $\gamma$ represents the efficiency of the site's "advertising technology", and we adopt the convention that a higher $\gamma$ corresponds to an advertising technology that is generally more efficient. Formally, we suppose that the function $\delta(\cdot, \cdot)$ is twice continuously differentiable, and, letting $\delta_{x}^{\prime}$ denote its derivative with respect to argument $x$, that it satisfies $\delta_{a}^{\prime}>0$ and $\delta_{a a}^{\prime \prime}>0$ 
as well as $\delta_{\gamma}^{\prime}<0$ and $\delta_{a \gamma}^{\prime \prime}<0$, for all $a>0$

Users are heterogeneous in their valuations, $v_{i}$, for the site's content, distributed according to a continuously differentiable pdf, $f(\cdot)$, strictly positive over some interval $[\underline{v}, \bar{v}]$, where $0 \leq \underline{v}<\bar{v}$. Any user who does not visit the site receives an "outside option" payoff parameterized by $\chi$. We define the site's demand function $D(\delta+\chi) \equiv \int_{\delta+\chi}^{\infty} f(x) d x$, which counts the number of users for whom the condition $v_{i}-\delta(a, \gamma) \geq \chi$ is satisfied. Note that $D(\cdot)$ is a twice differentiable and decreasing function. Let $h(x) \equiv \frac{D(x)}{-D^{\prime}(x)}$ denote the inverse hazard rate, which we assume to be decreasing: $h^{\prime}<0.6$ The site's profits, $\Pi$, can thus be written

$$
\Pi=(a-c) D(\delta(a, \gamma)+\chi)
$$

where $c \in \mathbb{R}$ denotes the cost per user of "serving" the site, which we assume to be constant.

A word on our motivation for not modeling the "two-sided" aspect of the website's profit maximization problem.7 The main reason for this is that, in subsequent sections, we are interested in isolating the distortions that arise from multiple websites' failure to coordinate with one another and want to avoid the added complication of frictions between the websites that show ads and the advertisers themselves. Note that our approach would be equivalent to assuming, in the well-known model of Anderson and Coate (2005), that the intermediaries (in their case television and radio broadcasters), can perfectly price discriminate to advertisers..$^{8}$ Indeed our framework does not preclude the possibility that advertising influences users' marginal valuation for the goods being advertised, as in Becker and Murphy (1993), so long as advertisers can perfectly price discriminate in their transactions with users.

\section{Equilibrium}

The site's profit maximization problem leads to a unique solution, $a^{*}$, which we assume to be strictly positive. It is characterized by the first-order condition

$$
a^{*}-c=\frac{h\left(\delta\left(a^{*}, \gamma\right)+\chi\right)}{\delta_{a}^{\prime}\left(a^{*}, \gamma\right)}
$$

\footnotetext{
${ }^{6}$ This condition, while more than sufficient to satisfy the website's second-order condition, tightly ensures that, in response to a uniform, downward shift in users valuations for content (see expression (2)), the website optimally decreases $a$, which we suspect is the most relevant case on which to focus. At the end of Section 3.1. we discuss the impact of relaxing this. Weyl and Fabinger (2009) provide a very broad analysis of the role of this class of assumption in monopoly and oligopoly pricing problems.

7 Gomes (2009) and White (2009) focus on two-sided aspects of search advertising.

${ }^{8}$ Unlike the present model, Anderson and Coate (2005) assume a linear advertising technology. Works that consider non-linear advertising technologies include Gabszewicz et al. (2004), Choi (2006) and Crampes et al. (2009).
} 
This variant of the classical monopoly pricing formula takes into account the rate at which an increase in ad revenue per user increases user distraction, captured by $\delta_{a}^{\prime}$. As in a standard monopoly pricing problem, the site trades off infra-marginal gain against marginal loss. Here, however, it is as if there were two "prices", $\delta$ and $a$. While the latter mediates the number of users who drop out at the margin, the former determines the website's infra-marginal gain.

To analyze the equilibrium outcome as a function of both the users' outside option and the efficiency of the site's advertising technology, we write $a^{*}=a^{*}(\chi, \gamma)$. First with respect to $\chi$, we have

$$
a_{\chi}^{* \prime}=\frac{h^{\prime}}{\delta_{a}^{\prime}\left(1-h^{\prime}\right)+\frac{h \delta_{a a}^{\prime \prime}}{\delta_{a}^{\prime}}}<0 .
$$

The optimal revenue per user decreases as the outside option gets more appealing. Moreover, since

$$
0<\frac{d\left\{\delta\left(a^{*}(\chi, \gamma)+\chi\right\}\right.}{d \chi}=\frac{h^{\prime}}{1-h^{\prime}+\frac{h \delta_{a a}^{\prime \prime}}{\delta_{a}^{\prime \prime}}}+1<1,
$$

equilibrium distraction also decreases with $\chi$ but at a rate low enough so as to provoke a decrease in demand.

Turning to the advertising technology, $\gamma$, we have

$$
a_{\gamma}^{* \prime}=-\frac{\Pi_{a \gamma}^{\prime \prime}}{\Pi_{a a}^{\prime \prime}}=-\frac{D^{\prime} \delta_{\gamma}^{\prime}+\frac{h}{\delta_{a}^{\prime}}\left(D^{\prime} \delta_{a \gamma}^{\prime \prime}+D^{\prime \prime} \delta_{a}^{\prime} \delta_{\gamma}^{\prime}\right)}{2 D^{\prime} \delta_{a}^{\prime}+\frac{h}{\delta_{a}^{\prime}}\left(D^{\prime} \delta_{a a}^{\prime \prime}+D^{\prime \prime} \delta_{a}^{\prime 2}\right)}>0 .
$$

In (4), the left-hand equality follows from the implicit function theorem, and the right-hand inequality follows from the hazard rate assumption. As the advertising technology becomes more efficient, the website aims to receive more revenue per user. While the website benefits from an improvement in the advertising technology, for users, such a change may in fact be harmful. Proposition 1 describes the conditions under which this is the case.

Proposition 1. As the advertising technology becomes more efficient, equilibrium distraction increases $(d \delta / d \gamma>0)$ if and only if

$$
\varepsilon_{\delta_{\gamma}^{\prime}}-\varepsilon_{\delta_{a}^{\prime}}>\frac{a^{*}}{a^{*}-c}
$$

where $\varepsilon_{\delta_{\gamma}^{\prime}} \equiv a^{*} \delta_{a \gamma}^{\prime \prime} / \delta_{\gamma}^{\prime}>0$ and $\varepsilon_{\delta_{a}^{\prime}} \equiv a^{*} \delta_{a a}^{\prime \prime} / \delta_{a}^{\prime}>0$.

Proof.

$$
\frac{d \delta}{d \gamma}=\delta_{a}^{\prime} a_{\gamma}^{* \prime}+\delta_{\gamma}^{\prime}>0
$$


Using (4), we have that the condition in (6) is equivalent to

$$
\begin{gathered}
\frac{\Pi_{a \gamma}^{\prime \prime}}{\delta_{\gamma}^{\prime}}<\frac{\Pi_{a a}^{\prime \prime}}{\delta_{a}^{\prime}} \\
\Leftrightarrow D^{\prime}+\frac{h}{\delta_{a}^{\prime}}\left(D^{\prime} \frac{\delta_{a \gamma}^{\prime \prime}}{\delta_{\gamma}^{\prime}}+D^{\prime \prime} \delta_{a}^{\prime}\right)<2 D^{\prime}+\frac{h}{\delta_{a}^{\prime}}\left(D^{\prime} \frac{\delta_{a a}^{\prime \prime}}{\delta_{a}^{\prime}}+D^{\prime \prime} \delta_{a}^{\prime}\right) \\
\Leftrightarrow \frac{h}{\delta_{a}^{\prime}}\left(\frac{\delta_{a \gamma}^{\prime \prime}}{\delta_{\gamma}^{\prime}}-\frac{\delta_{a a}^{\prime \prime}}{\delta_{a}^{\prime}}\right)>1
\end{gathered}
$$

Using (1) and rearranging gives the result.

The most intuitive way to understand the result in Proposition 1 is perhaps to let $\delta_{a \gamma}^{\prime \prime}<0$ vary, while holding fixed all other terms. When $\delta_{a \gamma}^{\prime \prime}$ is "very negative", then improvement in advertising technology brought on by an increase in $\gamma$ is such that it significantly lowers the marginal distraction caused by additional advertising. In this case, the website has an incentive to significantly increase the revenue it receives per user and thus to exclude more users than it did previously. On the other hand, when $\delta_{a \gamma}^{\prime \prime}$ is suffienciently "small", then the strength of the site's incentives to extract surplus from users is decreased, and users come away better off. As depicted in Figure 1 , in the former case, an increase in $\gamma$ corresponds to a clockwise rotation of the function $\delta(\cdot, \cdot)$, while, in the latter case, such a change corresponds to a shift downwards.

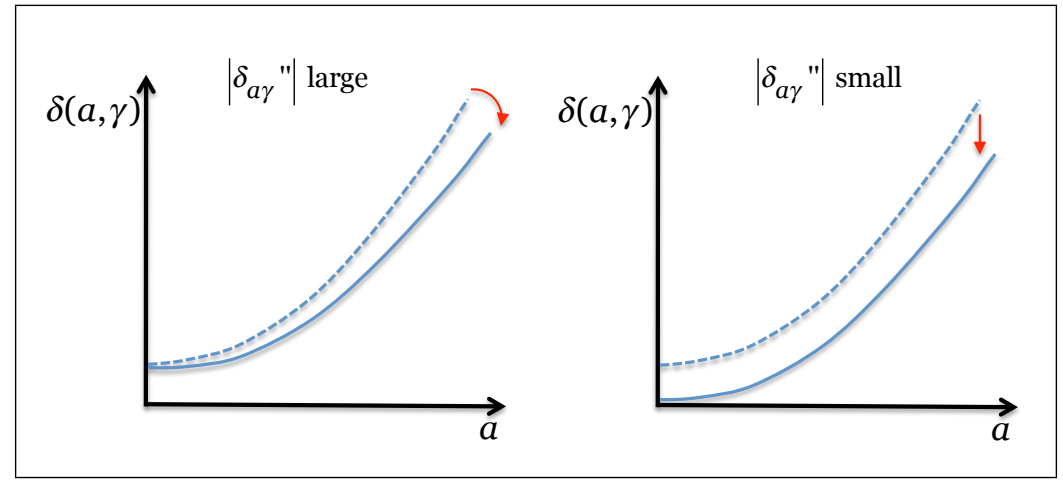

Figure 1: Two sorts of improvement in advertising technology

Briefly considering an example, let $\delta(a, \gamma)=g(\gamma) a^{\zeta}$, where $g^{\prime}(\gamma)<0<g(\gamma), \forall \gamma$, and where $\zeta>1$. Under these circumstances, $\delta_{a \gamma}^{\prime \prime} / \delta_{\gamma}^{\prime}-\delta_{a a}^{\prime \prime} / \delta_{a}^{\prime}=1 / a$ and thus $\varepsilon_{\delta_{\gamma}^{\prime}}-\varepsilon_{\delta_{a}^{\prime}}=1$. Hence, with advertising technologies in this family of functions, users strictly benefit from an increase in $\gamma$ if and only if the marginal cost of serving the site, $c$, is positive. 


\subsection{Alternative Specification of Advertising Technology}

There are also interesting economic issues that arise when comparing advertising technologies that are neither unambiguously more efficient nor unambiguously less efficient than one another. It seems reasonable to imagine that one method used to show ads could be less distracting than another in small quantities but that the annoyance it causes could accelerate more quickly. ${ }^{9}$ For instance, websites that display "splash pages" to visitors before showing them content seem likely to draw significant attention to these advertisements but also seem likely turn users away at a fast rate if they increase the imposition such ads cause. One might thus imagine that such a site's advertising technology starts off rather flat and then turns up steeply. On the other hand, websites that show "contextual" (text) ads seem likely have an advertising technology that is closer to being linear.

While our assumption on $\delta(\cdot, \cdot)$ that $\delta_{a \gamma}^{\prime \prime}$ is globally negative rules out the possibility of such a comparison using this function, we briefly consider one such example. Let the advertising technology be denoted by $\widetilde{\delta}(a, \gamma)=a^{\gamma}$, where $\gamma>1 \cdot{ }^{10}$ We further assume that $c, \chi=0$ and that $v_{i} \sim \mathrm{U}[0, \bar{v}]$. The site thus solves

$$
\max _{a} a\left(\frac{\bar{v}-a^{\gamma}}{\bar{v}}\right)
$$

which yields equilibrium advertising and distraction levels, $a^{*}(\gamma)$ and $\widetilde{\delta}\left(a^{*}(\gamma), \gamma\right)$, given by

$$
a^{*}(\gamma)=\left(\frac{\bar{v}}{1+\gamma}\right)^{1 / \gamma}, \quad \widetilde{\delta}\left(a^{*}(\gamma), \gamma\right)=\frac{\bar{v}}{1+\gamma}
$$

Users' consumer surplus is thus

$$
\frac{\bar{v}}{2}\left(\frac{\gamma}{1+\gamma}\right)^{2}
$$

Note that users are made unambiguously better off by an increase in $\gamma$, since this gives the site an incentive to cause less distraction. The site's profit's, $\Pi(\gamma)$, are

$$
\Pi(\gamma)=\frac{\gamma}{1+\gamma}\left(\frac{\bar{v}}{1+\gamma}\right)^{1 / \gamma}
$$

\footnotetext{
${ }^{9}$ One must, of course, take into account the caveat that, here, a "quantity" of advertisement corresponds to a level of expected revenue per user. For the sake of this discussion, it is useful to suppose that showing more ads translates into higher revenue per user for the site. The extent to which this is true is an interesting empirical issue.

${ }^{10}$ Gabszewicz et al. (2004) use this technology to derive the equilibrium "location choice" of competing television stations but do not discuss the intuition behind different values of $\gamma$.
} 
An increase in $\gamma$ can either help or harm the site, depending on the size of $\bar{v}$. As Figure 2 shows, when $\bar{v}$ is large, the site decreases its advertising level in response to an increase in $\gamma$ and vice versa when $\bar{v}$ is small.

To see why this is the case, hold $\gamma$ fixed and imagine an increase in $\bar{v}$. Note that the proportion of users that are served remains constant and is given by $\gamma /(1+\gamma)$. It thus follows that as $\bar{v}$ increases, so does $\widetilde{\delta_{a}^{\prime}}\left(a^{*}(\gamma), \gamma\right)$, the level of marginal distraction from advertising, evaluated at the equilibrium advertising level. When $\bar{v}$ takes a low value, $a^{*}$ falls in the zone where an increase in $\gamma$ makes the distraction curve locally flatter, whereas when $\bar{v}$ takes a high value, $a^{*}$ falls in the zone where an increase in $\gamma$ makes the distraction curve locally steeper.

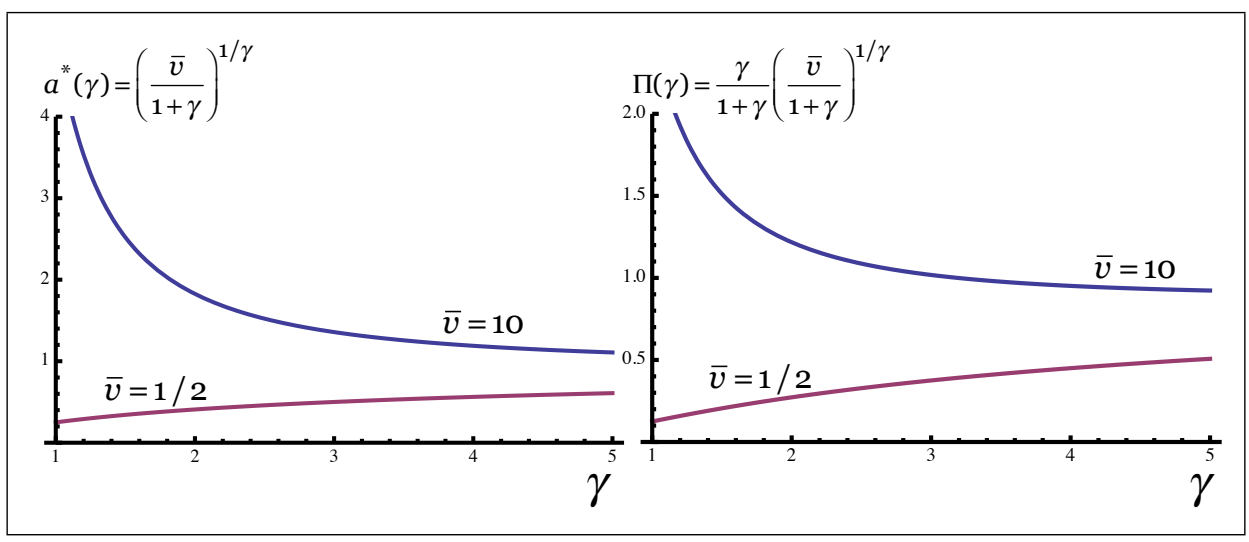

Figure 2: Equilibrium advertising level (left) and profits (right) as functions of $\gamma$, for two levels of $\bar{v}$, when the advertising technology is $\widetilde{\delta}=a^{\gamma}$

\section{Surfing Among Sites}

As discussed in the introduction, an essential feature of the Internet is the existence and availability to users of countless websites. In what we believe to be typical or, at least, very frequent episodes of browsing activity, users' fulfill one goal or need by surfing around among multiple sites. The most easily recognizable example of this may be the use of a search engine to help locate a site that contains a particular piece of content. While the precise details of surfing activity clearly vary greatly from one instance to another and from one user to another, our simple model identifies two phenomena that seem generally to be at play in such settings, in which different sites play a complementary role. These phenomena are the classical issue of double marginalization and a novel distortion we term mis-marginalization. 
To describe these, we generalize the model introduced in Section 2 in the following way. Suppose there are $n \geq 1$ websites, indexed by $j$, that, for users, are perfect complements. Let $a_{j}$ denote site $j$ 's advertising level, while $\boldsymbol{a} \equiv\left(a_{1}, \ldots, a_{j}, \ldots, a_{n}\right)$ denotes the vector of advertising levels, and $\bar{a} \equiv \sum_{j=1}^{n} a_{j}$ denotes the sum of the sites' advertising levels. We denote by $c_{j}$ the marginal cost of serving site $j$ and by $\bar{c} \equiv \sum_{j=1}^{n} c_{j}$ the sum of the sites' marginal costs. User distraction is given by $\delta(\boldsymbol{a})$, where $\delta: \mathbb{R}_{+}^{n} \rightarrow \mathbb{R}$. We assume that $\delta_{a_{j^{\prime}}}^{\prime} \delta_{a_{j} a_{j}}^{\prime \prime}>0, \forall a>0$. Finally, we assume that $\chi=0$, and thus demand for the bundle of sites is given by $D(\delta(\boldsymbol{a}))$.

As a benchmark, we consider the profit maximization problem of the entire set of websites, which we refer to as the "industry". We then compare the solution to this problem to the outcome when sites set their advertising levels non-cooperatively. The industry profit maximization problem is given by

$$
\max _{a}(\bar{a}-\bar{c}) D(\delta(\boldsymbol{a}))
$$

the solution to which, $a^{\Pi}$, is characterized by the set of first-order conditions

$$
\bar{a}^{\Pi}-\bar{c}=\frac{h\left(\delta\left(\boldsymbol{a}^{\Pi}\right)\right)}{\delta_{a_{j}}^{\prime}\left(\boldsymbol{a}^{\Pi}\right)}, \quad \forall j,
$$

where $\bar{a}^{\Pi}$ denotes the sum of the elements in $\boldsymbol{a}^{\Pi}$. Note that (7) implies that $\delta_{a_{j}}^{\prime}\left(\boldsymbol{a}^{\Pi}\right)=\delta_{a_{k}}^{\prime}\left(\boldsymbol{a}^{\Pi}\right)$, $\forall j, k$. Thus, in order for industry profits to be maximized, the marginal distraction from each site's advertising must be equalized.

We now examine sites' equilibrium behavior, using simultaneous Nash Equilibrium as our solution concept. Taking as given other sites' equilibrium advertising levels, $\boldsymbol{a}_{-j^{\prime}}^{*}$ site $j$ solves

$$
\max _{a_{j}}\left(a_{j}-c_{j}\right) D\left(\delta\left(a_{j}, a_{-j}^{*}\right)\right)
$$

yielding first-order condition

$$
a_{j}^{*}-c_{j}=\frac{h\left(\delta\left(\boldsymbol{a}^{*}\right)\right)}{\delta_{a_{j}}^{\prime}\left(\boldsymbol{a}^{*}\right)} .
$$

Summing over all sites' first-order conditions gives the following expression for the total margin per user the sites receive in equilibrium,

$$
\bar{a}^{*}-\bar{c}=h\left(\delta\left(\boldsymbol{a}^{*}\right)\right) \sum_{j=1}^{n} \frac{1}{\delta_{a_{j}}^{\prime}\left(\boldsymbol{a}^{*}\right)}
$$

The two distortions with respect to the industry optimum, double and mis-marginalization, can be appreciated by comparing equations (7) and (8). In particular, consider the right-most 
term in (8). If the sites were identical ${ }^{11}$ and the equilibrium in question were symmetric, we could write

$$
\sum_{j=1}^{n} \frac{1}{\delta_{a_{j}}^{\prime}\left(\boldsymbol{a}^{*}\right)}=\frac{n}{\delta_{a_{k}}^{\prime}\left(\boldsymbol{a}^{*}\right)}, \forall k
$$

The right-hand side of (9) captures, in isolation, the well-known double marginalization distortion, whereby each site balances its infra-marginal gain against only its own marginal loss and fails to take into account the externality it imposes on other sites.

In the general case, when the sites are not identical, the condition in (9) generically does not hold in equilibrium. When this condition fails to be satisfied, we say that there is mismarginalization. To be precise, mis-marginalization refers to the phenomenon whereby sites set advertising levels such that it would be feasible to increase total profits while holding fixed the total amount of distraction and thus serving the same number of users. While double marginalization results from the sites' failure to set the proper overall level of distraction, mis-marginalization is a separate coordination problem that results from the sites' failure to allocate the distraction they cause, or their demands for user attention, in the most efficient way.

\subsection{The Effects of Competition}

In situations where sellers of complementary components engage in double marginalization, a well known "solution" to this coordination problem, from the standpoint of total industry profits, is to increase competition in the markets for all but one of the components. For instance, suppose "hardware" and "software" were two perfectly complementary products, originally produced by two separate firms, the sum of whose prices is inefficiently high. If the market for hardware became perfectly competitive, then the single software maker would be in a position to charge a price that implements the optimum outcome for the industry as a whole ${ }^{12}$

In contrast, when a set of complementary websites, whose revenue comes exclusively from advertising, engage in both double and mis-marginalization, there can be a tradeoff between solving the former coordination problem and solving the latter. As a result, such an increase in competition no longer represents a solution from the standpoint of the industry as a whole. Moreover, it can, in fact, lead to a decrease in total profits.

\footnotetext{
${ }^{11}$ In this context, by sites being "identical" we mean that, in addition to sites having common marginal costs, $\delta(\cdot)$ is symmetric with respect to all of its arguments. Clearly, since the sites are perfect complements, they all face the same demand function.

${ }_{12}$ Casadesus-Masanell et al. (2007) and Cheng and Nahm (2007) study variations of such models, applied to these industries, focusing on the case where the producers in the sectors with competition are vertically differentiated.
} 
To see this, we specialize the model introduced earlier in this section to the case where there are two complementary sites. In line with the paper's lead example, we refer to the two sites as a "Search Engine" (SE) and a "Content Website" (CW). Let the SE be indexed by $s$ and the CW by $w$. We assume that the advertising technology is given by $\delta(\boldsymbol{a})=\delta_{s}\left(a_{s}\right)+\delta_{w}\left(a_{w}\right)$, where $\delta_{j}^{\prime}, \delta_{j}^{\prime \prime}>0, j=s, w$.

We now examine the effects of increasing the competition faced by one of the two sites, which we will assume to be the $\mathrm{CW} \mathrm{H}^{13}$ Define the function $\bar{\Pi}\left(a_{w}\right)$, denoting total industry profits as a function of the level of advertising chosen by the CW, assuming the SE bestresponds to the CW's choice. Formally,

$$
\bar{\Pi}\left(a_{w}\right) \equiv \Pi^{S E}\left(a_{s}^{*}\left(\delta_{w}\left(a_{w}\right)\right), \delta_{w}\left(a_{w}\right)\right)+\Pi^{C W}\left(a_{w}, \delta_{s}\left(a_{s}^{*}\left(\delta_{w}\left(a_{w}\right)\right)\right)\right)
$$

where $a_{s}^{*}\left(\delta_{w}\left(a_{w}\right)\right) \equiv \arg \max _{a_{s}} \Pi^{S E}\left(a_{s}, \delta_{w}\left(a_{w}\right)\right)$. Taking the derivative of (10) gives ${ }^{14}$

$$
\begin{aligned}
\bar{\Pi}^{\prime}\left(a_{w}\right)=\underbrace{\frac{\partial \Pi^{S E}}{\partial a_{s}} a_{s}^{* \prime} \delta_{w}^{\prime}+\frac{\partial \Pi^{S E}}{\partial \delta_{w}} \delta_{w}^{\prime}+}_{0}+\frac{\partial \Pi^{C W}}{\partial a_{w}}+\frac{\partial \Pi^{C W}}{\partial \delta_{s}} \delta_{s}^{\prime} a_{s}^{* \prime} \delta_{w}^{\prime} & \\
= & \underbrace{\left(a_{w}-c_{w}\right) D^{\prime} \delta_{w}^{\prime}\left(1+\delta_{s}^{\prime} a_{s}^{* \prime}\right)}_{\text {Marginal Effect }}+\underbrace{D\left(1-\frac{\delta_{w}^{\prime}}{\delta_{s}^{\prime}}\right)}_{\text {Infra-marginal Effect }} .
\end{aligned}
$$

The right-hand side of equation (11) separates the impact of a change in $a_{w}$ into a marginal effect and an infra-marginal effect of a change in $a_{w}$. Regarding the former, when $a_{w}$ increases, the propensity of users to stop visiting the combination of sites is mitigated by the corresponding reduction in distraction caused by the SE. Regarding the latter effect, when $a_{w}$ increases, CW revenue per user increases proportionally to the change in $a_{w}$; however, when the SE responds optimally, its revenue per user decreases by the ratio of the CW's marginal distraction to the SE's marginal distraction.

Stemming from this analysis, we now state Proposition 2. It shows the sub-optimality, to the industry as a whole, of both the configuration with one SE and one CW and the configuration with one SE and perfectly competitive CWs.

Proposition 2. (a) Starting from the Nash Equilibrium outcome between the search engine and the content website, if the CW advertising level, $a_{w}$, is exogenously decreased, and the SE responds

\footnotetext{
${ }^{13}$ In the subsequent analysis, one can interchange the roles of the SE and the CW.

${ }^{14}$ To derive the right-hand side of 111 , note that $\frac{\partial \Pi^{S E}}{\partial \delta_{w w}}=\left(a_{s}^{*}-c_{s}\right) D^{\prime}=-\frac{D}{\delta_{s}^{\prime}}, \frac{\partial \Pi^{C W}}{\partial a_{w}}=D+\left(a_{w}-c_{w}\right) D^{\prime} \delta_{w}^{\prime}$ and $\frac{\partial \Pi^{C W}}{\partial \delta_{s}}=\left(a_{w}-c_{w}\right) D^{\prime}$.
} 
optimally, then total industry profits increase.

(b) Starting from an outcome featuring the perfectly competitive level of CW advertising ( $a_{w}=c_{w}$ ) and monopolistic behavior by the $S E$, if $a_{w}$ is exogenously increased, then total industry profits increase if and only if $\delta_{w}^{\prime}\left(c_{w}\right)<\delta_{s}^{\prime}\left(a_{s}^{*}\left(\delta_{w}\left(c_{w}\right)\right)\right)$.

Proof. (a) To show the first claim, let $a_{w}^{N E}$ denote the CW's Nash Equilibrium advertising level. Since $D+\left(a_{w}^{N E}-c_{w}\right) D^{\prime} \delta_{w}^{\prime}=0$, and since $a_{w}^{N E}-c_{w}=h / \delta_{w}$, we have

$$
\bar{\Pi}^{\prime}\left(a_{w}^{N E}\right)=-D \cdot\left(\frac{\delta_{w}^{\prime}}{\delta_{s}^{\prime}}-\delta_{s}^{\prime} a_{s}^{* \prime}\right)>0,
$$

where (12) holds since (2) implies that $a_{s}^{* \prime}\left(\delta_{s}^{\prime}\right)=h^{\prime} /\left(\delta_{s}^{\prime}\left(1-h^{\prime}\right)+h \delta_{s}^{\prime \prime} / \delta_{s}^{\prime}\right)$. Plugging in the expression for $a_{s}^{* \prime}$ and rearranging reveals that (12) is equivalent to $\delta_{w}^{\prime}\left(1+h \delta_{s}^{\prime \prime} / \delta_{s}^{\prime 2}\right)>$ $h^{\prime}\left(\delta_{s}^{\prime}+\delta_{w}^{\prime}\right)$, which holds since all terms are positive except $h^{\prime}$.

(b) For the second claim, we have

$$
\bar{\Pi}^{\prime}\left(c_{w}\right)=D \cdot\left(1-\frac{\delta_{w}^{\prime}\left(c_{w}\right)}{\delta_{s}^{\prime}\left(a_{s}^{*}\left(\delta_{w}\left(c_{w}\right)\right)\right)}\right)
$$

and thus $\bar{\Pi}^{\prime}\left(c_{w}\right)>0 \Leftrightarrow \delta_{w}^{\prime}\left(c_{w}\right)<\delta_{s}^{\prime}\left(a_{s}^{*}\left(\delta_{w}\left(c_{w}\right)\right)\right)$.

Part (b) of Proposition 2 highlights the way in which mis-marginalization plays an important role in determining the optimal configuration for the overall industry. To see this, compare the current model with that of a "traditional" industry in which producers of complementary goods each charge consumers money in exchange for their respective components. In the latter case, consumers are indifferent about what fraction of their money goes to the respective producers. In contrast, with advertising, a unit of consumer attention translates at a variable rate to revenue for the website. As a result, the remedy for double marginalization of increased competition among $\mathrm{CW}$ can come at a cost. When the $\mathrm{CW}$ advertising technology is sufficiently efficient for low levels of revenue per user, a move to perfect competition among CWs, which alleviates the double marginalization problem, tends to exacerbate the problem of mis-marginalization.

To further illustrate this trade-off, we consider the following stylized game. In the first stage, the SE offers a contract to the CW, which the CW can either accept or reject. We assume that advertising levels are not precisely verifiable, and that it is possible for an outside enforcer to judge only whether the CW has set a level of advertising that is zero or positive. As a result, a feasible contract stipulates an amount of money, $T$, to be paid from 
the SE to the CW in exchange for the latter not to advertise. For simplicity, assume that the CW's marginal cost of serving the site is zero. Let $\Pi^{S E *}$ and $\Pi^{C W_{*}}$ denote the equilibrium profits if no contract is signed and let $\bar{\Pi}(0)$ denote the gross profits the SE receives if the CW accepts a contract and thus sets $a_{w}=0$.

In the game's Subgame Perfect Equilibrium, whenever $\bar{\Pi}(0) \geq \Pi^{S E *}+\Pi^{C W_{*}}$, the SE offers a payment of $T=\Pi^{C W_{*}}$, and the CW accepts. Otherwise, no acceptable offer is made. To identify circumstances under which each outcome occurs, suppose that the respective advertising technologies are given by $\delta_{s}\left(a_{s}\right)=\left(a_{s} / \sigma\right)^{2}$ and $\delta_{s}\left(a_{w}\right)=\left(a_{w} / \omega\right)^{2}$, where $\sigma, \omega>0$, and that $v_{i} \sim \mathrm{U}[0, \bar{v}]$. Under these assumptions, we find $\Pi^{S E *}=\sigma \sqrt{\bar{v}} / 4$ and $\Pi^{C W_{*}}=\omega \sqrt{\bar{v}} / 4$, while $\bar{\Pi}(0)=2 \sqrt{\bar{v}} / 3^{3 / 2}$. This leads to the result that $\Pi^{S E *}+\Pi^{C W *}>\bar{\Pi}(0)$, whenever the (approximate) condition $\omega / \sigma>3 / 5$ is satisfied. In such circumstances, the SE does not offer an acceptable contract $t^{15}$ Correspondingly, when $\omega / \sigma>3 / 5$ is satisfied, if the CW "sector" went from being monopolistic to perfectly competitive, then total industry profits would decrease.

\section{The Impact of Competition on Users}

Users directly perceive the effects of double marginalization but are indifferent to mismarginalization. Consequently, they unambiguously benefit from an increase in the level of competition in one of the sectors. Suppose CW advertising is at an arbitrary level, $a_{w}$, and that the SE best-responds by setting $a_{s}=a_{s}^{*}\left(\delta_{w}\left(a_{w}\right)\right)$. Expression (3) implies that, when $a_{w}$ decreases, so does the total level of distraction, and thus more users visit the combination of sites. Note, however, that this result is sensitive to the hazard rate assumption that $h^{\prime}<0$. Holding fixed other elements of the model, if the distribution of user valuations for the content were warped so as to locally violate this assumption, then, at such points, advertising levels would be strategic complements for the $\mathrm{CW}$ and the SE.

The following questions also arise, regarding the impact of competition on users, the answers to which are somewhat more ambiguous. If, starting from a configuration with one $\mathrm{SE}$ and one $\mathrm{CW}$, it were possible to increase competition in one of the two sectors, in which sector would users prefer for this to occur? Moreover, if such a change were to occur in one of the two sectors, would the one preferred by users coincide with the one preferred by the industry as a whole?

The result of Proposition 1 and the discussion in Section 2.1 serve as guides to answering these questions. The basic message that these give is that both users and the industry have a preference for advertising to be done using a technology that provides low levels

\footnotetext{
${ }^{15}$ One might note that, in this particular example, when $\omega / \sigma>3 / 5$ is satisfied, there would be an incentive for the CW to offer a contract to the SE not to advertise.
} 
of distraction. However, users have a preference for technologies with a high degree of curvature, since they give sites incentive to distract less. On the other hand, holding the level of distraction fixed, websites prefer technologies with a low degree of curvature, since these allow them to increase their revenue from loyal (infra-marginal) users while turning away relative few indifferent (marginal) users.

\section{Entry and Competition with Market Coverage}

In this section, we address additional effects that arise when there are two types of sites that users find to be complementary. Specifically, assuming there is a single SE, we examine the (i) impact of changes in both the number of CWs and the degree to which they are horizontally differentiated, and (ii) the incentives for CWs to enter the market, comparing equilibrium with the social optimum. As it helps to uncover effects not already discussed in Section 3, we consider a model in which there is complete market coverage and consider the impact of this aspect on the model's behavior. A notable result when there is complete market coverage is that tougher competition among content websites harms users.

The model in this section is a variant of the "circular city" model introduced by Salop (1979). Content websites are "located" equidistant from one another. Users visit the search engine, which shows them a set of content websites on a results page. Users then choose to visit the content site that best corresponds to their tastes.

The section proceeds as follows. First, we describe the model. Second we discuss the socially optimal outcome, after which we analyze the equilibrium. We consider both the case with an exogenous number of content websites and the case where their number is determined endogenously by a free entry condition.

\subsection{The Setup}

There is one SE, an arbitrarily large number of CWs and a continuum of users of mass one. Users seek to obtain or consume some form of content that is available from CWs and must use the search engine to locate the set of candidate sites. If a user $i$ searches and then visits content website $w$, she receives a payoff of

$$
v-\left|x_{i}-x_{w}\right| \tau-\delta_{s}\left(a_{s}\right)-\delta_{w}\left(a_{w}\right)
$$

where $v>0$ is common to all users, the "distance", $\left|x_{i}-x_{w}\right|$, represents the discrepancy, measured with respect to the rest of the $\mathrm{CW}$ market, between user $i$ 's ideal form for the 
content to take and the form in which it is presented by site $w$. The parameter $\tau$ captures the overall level of differentiation in the CW market. As before, $\delta_{s}\left(a_{s}\right)$ and $\delta_{w}\left(a_{w}\right)$ are the distraction from advertising on the search engine and on content website $w$. We assume that, as in the example of Section 3.1, $\delta_{s}\left(a_{s}\right)=\left(a_{s} / \sigma\right)^{2}$ and that, for all $w, \delta_{w}\left(a_{w}\right)=\left(a_{w} / \omega\right)^{2}$, where $\sigma, \omega>0$. We normalize users' outside option, $\chi$, to be zero.

We assume that the SE as well as all CWs serve their sites at zero marginal cost. SE profits are thus equal to advertising revenue per user, multiplied by the number of users and can be written as $a_{s} \times D_{s}\left(a_{s}, \boldsymbol{a}_{w}\right)$, where $\boldsymbol{a}_{w}$ denotes the vector of CW advertising levels. CW profits also include the fixed cost of entry, $K>0$, and can be written as $a_{w} \times D_{w}\left(a_{s}, a_{w-1}, a_{w}, a_{w+1}\right)-K$, when site $w$ enters and 0 otherwise.

We assume the following timing of the game. Note that in contrast to the approach of Section 3, in which we focused on simultaneous Nash Equilibria, here we assume a Stackelberg organization. The latter leads to a unique Subgame Perfect Equilibrium, while, under simultaneous Nash, there is a continuum of equilibria that create expositional inconvenience with little added insight.

1. Content websites decide whether or not to enter. We denote the number that do by $n \sqrt[16]{16}$

2. The search engine sets its advertising level, $a_{s}$.

3. Content websites that have entered set their advertising levels, $a_{w}, w=1, \ldots, n$.

4. Users decide either to visit the search engine and a content website of their chosing or to do nothing.

\subsection{Social Optimum}

Full market coverage is socially optimal if and only if $v \geq \sigma^{2} / 4+\omega^{2} / 4+\sqrt{\tau K} / 4$, and in this subsection we assume this condition is satisfied. Consequently, all users search and visit the CW located nearest them on the circle. Moreover, CWs $w=1 . . n$ all set a common advertising level, $\widehat{a_{w}}$. Given this, we can write total welfare as

$$
\underbrace{a_{s}}_{\text {SE profits }}+\underbrace{n\left(\frac{\widehat{a_{w}}}{n}-K\right)}_{\text {Total CW profits }}+\underbrace{v-\left(\frac{a_{s}}{\sigma}\right)^{2}-\left(\frac{\widehat{a_{w}}}{\omega}\right)^{2}-\frac{\tau}{4 n}}_{\text {User Surplus }} .
$$

\footnotetext{
${ }^{16}$ As it does not affect our results in any interesting way, we ignore the "integer constraint" when examining the number of firms. Mankiw and Whinston (1986) discuss the role of such a constraint in a model of entry.
} 
The socially optimal advertising levels can be found by taking the first-order conditions of (13) with respect to $a_{s}$ and $\widehat{a_{w}}$, yielding optimal values $a_{s}^{o}=\sigma^{2} / 2$ and $a_{w}^{o}=\omega^{2} / 2$. Thus a social planner chooses to expose users to advertisement from each site up to the point where its marginal distraction to the users exceeds the marginal revenue it brings to the site. The more efficient a particular type of site's advertising technology, reflected by $\sigma$ for the SE and by $\omega$ for the CWs, the more ads a social planner places on that type of site. As a result, SE profits increase with $\sigma, \mathrm{CW}$ profits increase with $\omega$, and consumer surplus decreases with both.

For a given number of CWs, $n$, total welfare is thus equal to

$$
\frac{\sigma^{2}}{4}+\frac{\omega^{2}}{4}-n K+v-\frac{\tau}{4 n}
$$

The fact that (14) increases with $\sigma$ and $\omega$ reflects the fact that society as a whole stands to gains from improvements in advertising technology. Within the model, this is because such improvements create more opportunities for attention to be efficiently transformed into advertiser revenue. Beyond the literal representation of the model, the behavior of this function reflects two phenomena. First, when the presentation of ads is more tactful, consumers of content can both enjoy the content they are interested in and respond to the advertisement without these activities conflicting with one another. Second, when the match between ads and users is more relevant, advertisers have a higher chance of profitably interacting with users and thus are willing to pay the sites more to have their ads appear. One may think of improvements, for a given type of site, in dimensions such as these as corresponding to increases in the site's efficiency parameter.

Turning to the number of CWs, $n$, first note that, when a social planner sets advertising levels, users benefit from having more CWs, while an increase in the level of differentiation, $\tau$, causes them harm. Both of these stem straightforwardly from the fact that either an increase in $n$ or a decrease in $\tau$ causes the content an average user consumes to be closer to her ideal. The socially optimal number of CWs solves the tradeoff between these benefits for users with the setup cost, $K$, incurred by each $\mathrm{CW}$.

The solution to this problem is found by maximizing (14) with respect to $n$, which gives the result $n^{o}=\sqrt{\tau / K}$. Plugging the optimal values $a_{s}^{o}, a_{w}^{o}$ and $n^{o}$ into, (13), the expression for total welfare, gives

$$
\underbrace{\frac{\sigma^{2}}{2}}_{\text {SE profits }}+\underbrace{\frac{\omega^{2}}{2}-\sqrt{\tau K}}_{\text {Total CW profits }}+\underbrace{v-\frac{\sigma^{2}}{4}-\frac{\omega^{2}}{4}-\frac{\sqrt{\tau K}}{4}}_{\text {User Surplus }} .
$$


Thus, at the social optimum, the SE makes positive profits, while the CWs may make positive or negative profits.

\subsection{Equilibrium}

We now analyze the subgame-perfect equilibrium of the game, assuming that, in equilibrium, the market is fully covered ${ }^{17}$ We begin by deriving the demand faced by content website $w$. We look for symmetric equilibria and denote the advertising level set by other CWs by $a_{-w}$. Define $\widetilde{v}\left(a_{s}\right) \equiv v-\left(\frac{a_{s}}{\sigma}\right)^{2}$. Demand for CW $w$ is then

$$
D_{w}\left(a_{s}, a_{w}, a_{-w}\right)=\left\{\begin{array}{cc}
\frac{1}{\tau}\left(\frac{\tau}{n}+\left(\frac{a_{-w}}{\omega}\right)^{2}-\left(\frac{a_{w}}{\omega}\right)^{2}\right), & \text { if }\left(\frac{a_{w}}{\omega}\right)^{2} \leq 2 \widetilde{v}\left(a_{s}\right)-\frac{\tau}{n}-\left(\frac{a_{-w}}{\omega}\right)^{2} \\
\frac{2}{\tau}\left(\widetilde{v}\left(a_{s}\right)-\left(\frac{a_{w}}{\omega}\right)^{2}\right), & \text { otherwise }
\end{array},\right.
$$

and CW $w$ 's profits are $a_{w} \times D_{w}\left(a_{s}, a_{w}, a_{-w}\right)$. Straightforward calculations yield the function $a_{w v}^{*}\left(a_{s}\right)$, mapping from the SE's level of advertising to the CWs' level of advertising in the continuation equilibrium, which is given by

$$
a_{w}^{*}\left(a_{s}\right)=\left\{\begin{array}{cc}
\omega \sqrt{\frac{\widetilde{v}\left(a_{s}\right)}{3}}, & \text { if } \widetilde{v}\left(a_{s}\right)<\frac{3 \tau}{4 n} \\
\omega \sqrt{\widetilde{v}\left(a_{s}\right)-\frac{\tau}{2 n}}, & \text { if } \frac{3 \tau}{4 n} \leq \widetilde{v}\left(a_{s}\right)<\frac{\tau}{n} \\
\omega \sqrt{\frac{\tau}{2 n}}, & \text { if } \frac{\tau}{n} \leq \widetilde{v}\left(a_{s}\right)
\end{array} .\right.
$$

Examining expression (15) gives the following insight. The nature of the game's equilibrium - i.e., whether the market turns out to be covered or uncovered - is determined by the search engine when it sets $a_{s}$. If it sets $a_{s}$ such that $\widetilde{v}\left(a_{s}\right)<3 \tau / 4 n$, then subsequent moves by the CWs will leave the market uncovered, whereas if $\widetilde{v}\left(a_{s}\right) \geq 3 \tau / 4 n$, then continuation play by the CWs will cover the market.

Turning to the previous stage of the game, in which the SE sets $a_{s}$, we derive the search engine's demand function. Using the CW's reaction function given in (15), the search engine's demand can be written as

$$
D_{s}\left(a_{s}\right)=\left\{\begin{array}{cc}
1, & \text { if }\left(\frac{a_{s}}{\sigma}\right)^{2} \leq v-\frac{3 \tau}{4 n} \\
\frac{4 n}{3 \tau}\left(v-\left(\frac{a_{s}}{\sigma}\right)^{2}\right), & \text { otherwise }
\end{array} .\right.
$$

\footnotetext{
${ }^{17}$ For the market to be fully covered in equilibrium, the necessary and sufficient condition is $v \geq$ $9 / 2(K \tau / 4 \omega)^{2 / 3}$. Note that when this condition fails, this model behaves in the same way as the class of models with one SE and one CW, discussed in Section 3 , with the exception that, here, we assume Stackelberg timing.
} 
Maximizing search engine profits, $a_{s} \times D_{s}\left(a_{s}\right)$, yields the following solution:

$$
a_{s}^{*}=\left\{\begin{array}{cc}
\sigma \sqrt{v-\frac{3 \tau}{4 n}}, & \text { if } v \geq \frac{9 \tau}{8 n} \\
\sigma \sqrt{\frac{v}{3}}, & \text { if } v<\frac{9 \tau}{8 n}
\end{array} .\right.
$$

As our initial assumption on the parameter values insures that $v \geq 9 \tau / 8 n$, we have that $a_{s}^{*}=\sigma \sqrt{v-3 \tau / 4 n}$. This expression has an interesting property: it is largest value of $a_{s}$ that the search engine can choose while still inducing the CWs to leave the market covered in the subsequent stage. The fact that the search engine chooses such a value is quite intuitive. If it increased its advertising level to $a_{s}^{*}+\epsilon$, then it would pass the kink in its demand function and lose users at a relatively fast rate, due both to users' distaste for additional search advertisement and to the fact that this would induce CWs to advertise more, further turning away users. On the other hand, if the SE decreased its advertising level, this would induce CWs to advertise more, leaving the market covered and maintaining the same consumption pattern among users.

\section{Results with an Exogenous Number of CWs}

We now analyze the outcome of the game, taking as given, $n$, the number of CWs. We first draw attention to the following result in Proposition 3.

Proposition 3. User surplus decreases when competition among content websites toughens, either as a result of an increase in their number, $n$, or as a result of an increase in $\tau$, the horizontal differentiation parameter.

Proof. Using the values found above, we can write total welfare as

$$
\underbrace{\sigma \sqrt{v-\frac{3 \tau}{4 n}}}_{\text {SE profits }}+\underbrace{\frac{\omega}{2} \sqrt{\frac{\tau}{n}}-n K}_{\text {Total CW profits }}+\underbrace{\frac{\tau}{4 n}}_{\text {User Surplus }},
$$

and the term for user surplus increases with $\tau$ and decreases with $n$.

This result stems not from any direct benefit that users derive from increased differentiation or from any direct harm they incur from an increase in the number of CWs. Instead, it is the SE's reaction to such changes that drives the result. When $n$ increases or $\tau$ decreases, the search engine anticipates that, in the final stage of the game, CWs will compete more fiercely 
with one another ${ }^{18}$ As a result of this heightened competition, the SE is able to advertise more while still inducing an equilibrium where the market is barely covered. Another way to see this is to think about the effects of such a change on the infra-marginal users - an increase in competition, in effect, makes them less different from marginal users than they were before, and since marginal users continue to receive a payoff of zero, infra-marginal users fare less well.

Having understood the nature of this interplay between the single SE and the multiple CWs, it is straightforward to see how a toughening of competition affects each type of site's fortunes. For the search engine, tougher competition among CWs is good. Meanwhile for total CW profits, both a decrease in $\tau$ and an increase in $n$ lower profits, the latter through an additional channel, since it increases $\mathrm{CWs}^{\prime}$ total fixed costs.

Note that, provided firms behave non-cooperatively when setting their advertising levels, the optimal number of firms, $n^{\circ *}$, can be found by maximizing (16) with respect to $n$, which gives the first-order condition

$$
\frac{3 \sigma \tau}{8\left(n^{o *}\right)^{2} \sqrt{v-\frac{3 \tau}{4 n^{\circ *}}}}-\frac{\tau \omega}{4\left(n^{\circ *}\right)^{2} \sqrt{\frac{\tau}{n^{* *}}}}-K-\frac{\tau}{4\left(n^{o *}\right)^{2}}=0 .
$$

While this equation does not admit a tractable closed-form solution for $n^{o *}$, we use it in the next subsection to compare this (constrained) optimal level of entry with the equilibrium level of entry.

\section{Free Entry of CWs}

We now study the equilibrium of the entire game, including the first stage in which $n^{*}$, the number of content websites, is determined endogenously. Setting an individual CW's profits equal to zero and solving yields

$$
n^{*}=\left(\frac{\omega}{2 K}\right)^{2 / 3} \tau^{1 / 3}
$$

None of the derivatives of this expression with respect to the parameters are surprising. Entry increases with both $\omega$, the efficiency with which a CW transforms attention into revenue and with $\tau$, the horizontal differentiation among websites. Meanwhile, entry decreases with $K$, the fixed cost of setting up a CW.

Regarding the efficiency of this outcome, there are two closely related questions of

\footnotetext{
${ }^{18}$ Note that this result does not depend on the Stackleberg timing whereby the SE moves before the CWs. The same phenomenon occurs when the two types of sites move simultaneously.
} 
particular relevance. First, provided that sites behave non-cooperatively when setting their advertising levels in the post-entry stages, how many CWs enter compared to the number that would optimize total welfare? Second, given that in equilibrium $\mathrm{CWs}^{\prime}$ enter until they are left with no profits, what values of the parameters maximize welfare for the remaining agents - the SE and the users? We address these issues in Propositions 4 and 5.

Proposition 4. When search engine advertising is sufficiently efficient, fewer content websites enter in equilibrium than would be socially optimal; when search engine advertising is inefficient enough, too few CWs enter in equilibrium.

Proof. For any $n^{*} \geq 1$, there exists $\sigma^{*}$ such that, (a) if $\sigma>\sigma^{*}$, the left-hand side of equation (17), with $n^{*}$ in place of $n^{o *}$, is strictly positive, and (b) if $\sigma<\sigma^{*}$, the left-hand side of equation (17), with $n^{*}$ in place of $n^{\text {o* }}$, is strictly negative.

This feature of the model stems, in part, from the logic explained in the discussion of socially optimal advertising levels, following expression (14), namely that a high value of $\sigma$ corresponds to high potential to create search engine profits at a low marginal cost to users. In the context of the free entry equilibrium, the entrance of an additional $\mathrm{CW}$, beyond $n^{*}$, emits a positive externality on the SE while emitting negative externalities on both the other CWs and on users (not to mention a negative "internality" on itself). Upon entering, the $\left(n^{*}+1\right)^{\text {th }} \mathrm{CW}$ brings about tougher competition among the other content websites, which induces the SE to raise its advertising level, causing a net loss to infra-marginal users. This positive externality benefiting the SE exceeds the negative externalities afflicting the CWs and the users when the SEs advertising is effective enough. We now turn to equilibrium comparative statics, which Proposition 5 describes and Figure 3 depicts.

Proposition 5. Equilibrium user surplus increases both with, $\tau$, the differentiation among content websites and with $K$, the fixed cost for $C W$ s to enter; it decreases with $\omega, C W$ advertising efficiency. Search engine profits are a decreasing function of user surplus. Moreover, if $v \geq 27 / 4 \sigma^{2}$, then changes leading to greater consumer surplus also lead to greater total welfare.

Proof. Combining (18) and (16), we find

$$
\underbrace{\sigma \sqrt{v-3\left(\frac{K \tau}{4 \omega}\right)^{2 / 3}}}_{\Pi^{S E}\left(\sigma, v, U\left(\frac{K \tau}{\omega}\right)\right)}+\underbrace{\left(\frac{K \tau}{4 \omega}\right)^{2 / 3}}_{U\left(\frac{K \tau}{\omega}\right)} .
$$

Furthermore, $\frac{\partial \Pi^{S E}}{\partial U}+1>0$ if $v \geq 27 / 4 \sigma^{2}$, since $\frac{\partial \Pi^{S E}}{\partial U}+1>0$ whenever $v>3 U\left(\frac{K \tau}{\omega}\right)+\left(\frac{3 \sigma^{2}}{2}\right)^{2}$, and, from assuming market coverage, we have $v \geq \frac{9}{2} U\left(\frac{K \tau}{\omega}\right)$. 


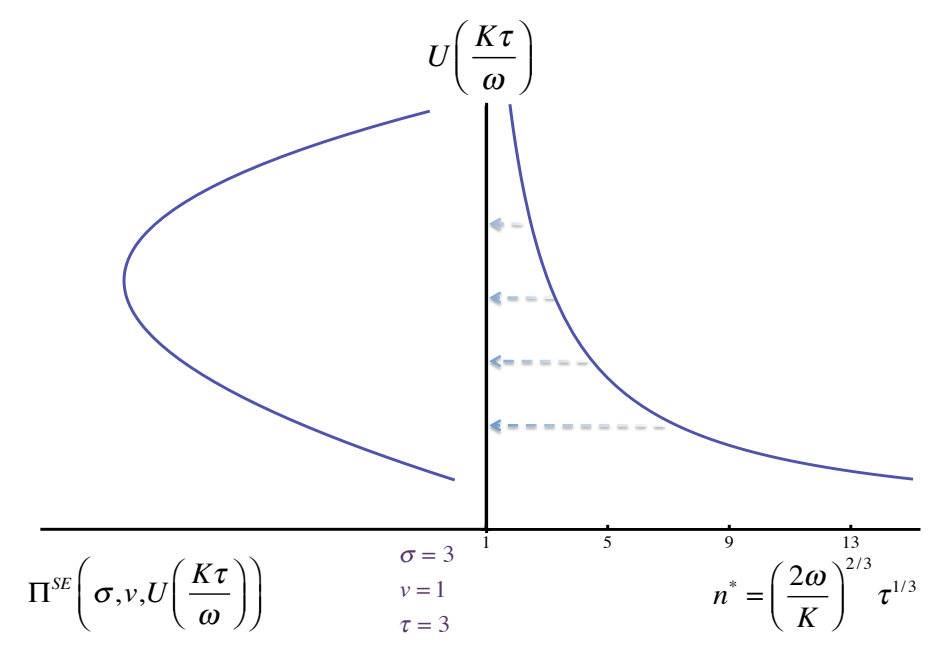

Figure 3: To the right of the vertical axis, user surplus appears as function of the $n^{*}$, the number of CWs entering in equilibrium. To the left, total welfare is plotted on the horizontal axis, as a function of user surplus.

Given free entry of CWs, the search engine and the users' interests are perfectly opposed to one another. It is important to bear in mind that in this model, all users are served in equilibrium, and, as a result, the issue of double marginalization is absent. We have thus isolated an effect of competition among CWs that, when allowed to increase orthogonally to the level of total distraction, harms users.

Considering this effect in a broader context, it shows that, when a single advertiser such as the SE and multiple advertisers such as the CWs provide users with access to content in a complementary way, there are two effects on users that can push in opposite directions. First, when competition among CWs increases and their advertising causes less distraction, if, in response, the SE increases its distraction at a rate that allows for total demand to expand, then there is a benefit of competition to users. On the other hand, the second possible effect of increased CW competition, emphasized in this sections' model, is that, holding the level of demand fixed, increased differentiation helps all users by widening the gap between those that are infra-marginal and those that are marginal.

\section{Conclusion}

In this paper, we have studied the implications of two important features of advertising on the Internet. The first feature is the wide variability in the efficiency of different websites' 
advertising technologies. The second feature is the complementary role that, under many circumstances, multiple sites play in an Internet user's browsing experience. We argue that a prime scenario when this complementarity is at play is when users visit a search engine in order to help locate a particular piece of content available on some other site.

To analyze these implications, we develop a model of advertising in which the "price" users pay to visit otherwise free websites is the "attention" they spend or the "distraction" they incur from being subjected to advertisement. In three main sections, we consider three main sets of issues. First, we analyze the circumstances under which users benefit from improvements in websites' advertising technology. Second, we identify two fundamental distortions - double and mis-marginalization - that arise when sites set their advertising policies non-cooperatively. Finally, we consider the case where a monopolist search engine serves all users and examine the effects of differentiation by websites that serve content as well as their incentives to enter the market. 


\section{References}

Anderson, Simon P. and Stephen Coate (2005), "Market Provision of Broadcasting: A Welfare Analysis." The Review of Economic Studies, 72, 947-972, URL http://www . jstor. org/stable/3700696.

Anderson, Simon P. and André de Palma (2009), "Competition for Attention in the Information (Overload) Age." Mimeo., URL http://www .virginia.edu/economics/Workshops/ papers/anderson/butter090401.pdf.

Becker, Gary S. and Kevin M. Murphy (1993), "A Simple Theory of Advertising as a Good or Bad." The Quarterly Journal of Economics, 108, 941-964, URL http://www. jstor .org/ stable/2118455.

Casadesus-Masanell, Ramon, Barry J. Nalebuff, and David Yoffie (2007), “Competing Complements." Mimeo., URL http://ssrn.com/abstract=1032461

Cheng, Leonard K. and Jae Nahm (2007), "Product Boundary, Vertical Competition, and the Double Mark-Up Problem." The RAND Journal of Economics, 38, 447-466, URL http: //www.jstor.org/stable/25046315.

Choi, Jay Pil (2006), "Broadcast Competition and Advertising with Free Entry: Subscription vs. Free-to-Air." Information Economics and Policy, 18, 181 - 196, URL http://dx. doi .org/ $10.1016 / j$.infoecopol.2005.12.002.

Cournot, Augustin (1838), Researches into the Mathematical Principles of the Theory of Wealth (N.T. Bacon trans.), 1971 edition. Augustus M. Kelley, New York.

Crampes, Claude, Carole Haritchabalet, and Bruno Jullien (2009), "Advertising, Competition and Entry in Media Industries." Journal of Industrial Economics, LVII, 7-31, URL http://dx.doi.org/10.1111/j.1467-6451.2009.00368.x.

Evans, David S. (2008), "The Economics of the Online Advertising Industry." Review of Network Economics, 7, 359-391, URL http://dx.doi.org/10.2202/1446-9022.1154.

Evans, David S. (2009), "The Online Advertising Industry: Economics, Evolution, and Privacy." Journal of Economic Perspectives, 23, 37-60, URL http://dx. doi.org/10.1257/ jep.23.3.37.

Gabszewicz, Jean J., Didier Laussel, and Nathalie Sonnac (2004), "Programming and Advertising Competition in the Broadcasting Industry." Journal of Economics \& Management Strategy, 13, 657-669, URL http://dx.doi.org/10.1111/j.1430-9134.2004.00027.x.

Gomes, Renato (2009), "Mechanism Design in Two-Sided Markets: Auctioning Users." Mimeo., URL http://www. depot.northwestern.edu/ rdg817/paper1.pdf.

Katona, Zsolt and Miklos Sarvary (2008), "Network Formation and the Structure of the Commercial World Wide Web." Marketing Science, 27, 764-778, URL http://mktsci. journal.informs.org/cgi/content/abstract/27/5/764. 
Mankiw, N. Gregory and Michael D. Whinston (1986), "Free Entry and Social Inefficiency." The RAND Journal of Economics, 17, 48-58, URL http://www. jstor .org/stable/ 2555627.

Rey, Patrick and Jean Tirole (1986), "The Logic of Vertical Restraints." The American Economic Review, 76, 921-939, URL http://www. jstor .org/stable/1816460.

Salop, Steven C. (1979), "Monopolistic Competition with Outside Goods." The Bell Journal of Economics, 10, 141-156, URL http://www. jstor.org/stable/3003323.

Weyl, E. Glen and Michal Fabinger (2009), "Pass-through as an Economic Tool." Mimeo., URL http://www. people.fas.harvard.edu/\%7Eweyl/Pass-through_10_09.pdf.

White, Alexander (2009), "Search Engines: Left Side Quality versus Right Side Profits." Mimeo., URL http://white.alex.online.fr/Home/Research_files/WhiteSE08.pdf. 